\title{
Psychosocial, psychiatric, and clinical implications of diabetic foot ulceration: A prospective analysis
}

\author{
Bala Neeru, Kaur Gagandeep ${ }^{1}$, Attri Joginder Pal ${ }^{1}$, Sukhminder Jit Singh Bajwa ${ }^{2}$, Sawney Harbandna, Kumar Rajesh ${ }^{3}$ \\ Departments of Psychiatry, ${ }^{1}$ Anaesthesia and ${ }^{3}$ Orthopedics, Government Medical College, Amritsar, ${ }^{2}$ Department of Anaesthesiology and Intensive \\ Care, Gian Sagar Medical College and Hospital, Ram Nagar, Banur, Punjab, India
}

\section{A B S T RAC T}

Background: Foot ulceration is very common complication of diabetes. Prevalence of psychiatric disorders is considered to be more in patients suffering from diabetic foot ulcers. Aim: The present study was carried out to observe and evaluate the various psychiatric disorders experienced by patients with diabetic foot ulceration, management of these disorders, and the metabolic abnormalities associated with the use of antipsychotic drugs. Materials and Methods: In this observational study, 293 patients in the age group of 25-70 years of either sex, who visited this hospital for the management of diabetic foot ulcers, were included after taking informed consent. They were prospectively followed for a period of 24 months. A phenomenological approach using semi-structured, conversational interviews was used. They were interviewed for the type of diabetes, duration of diabetes and duration of foot ulcer and other complications, type of treatment for diabetes and diabetic foot, psychosocial effects of foot ulceration on their daily The Hamilton rating scale for depression, questionnaire was used for assessing depression while the Hamilton anxiety rating scale questionnaire was used to rate the severity of anxiety. The data collected and analyzed for the type of psychiatric disorder, treatment options, type of drugs given and any side effects or complications occur as a result of these drugs. Descriptive statistical methods were used to analyze the data. Conclusion: Diabetic foot ulcer is associated with an increased incidence of various psychiatric disorders, depression being the most common among these. Drugs used to treat depression and associated with metabolic disturbances. Hyperglycemia associated with the use of antidepressants and antipsychotics further worsens the foot ulceration. Coordination between different specialties is important to manage the clinical, biological, and psychosocial aspects of this critical illness.

Key words: Anxiety, depression, diabetic foot ulcer, psychiatric aspects

\section{INTRODUCTION}

In India, approximately 42 million people are suffering from diabetes mellitus. Among them, approximately $15 \%$ develop foot ulcers as a complication of diabetes during their lifetime. ${ }^{[1]}$ Foot ulceration is a common complication of uncontrolled diabetes which develops either due to symmetrical polyneuropathy of sensory, motor and autonomic nerves, or micro-vascular

\begin{tabular}{|l|l|}
\hline \multicolumn{2}{|c|}{ Access this article online } \\
\hline Quick Response Code: & Website: \\
\hline & www.joshd.net \\
\hline & \\
\hline & \\
\hline
\end{tabular}

angiopathy. ${ }^{[2,3]}$ Diabetic foot ulcer is the second most common cause of lower limb amputation after traumatic amputation in India. ${ }^{[4]}$ As it is a chronic illness with no clearly defined pattern of onset and progression, management of this condition needs patient participation besides the ongoing medical treatment. Patients have to bring certain changes in their lifestyle which can impose a psychological burden on them. In developing countries like India, due to various socio-cultural, economic, and behavioral factors, there is a lack of awareness about the preventive measures as well as management of this disease. This further leads to poor compliance and adherence to treatment. Once foot ulcer develops, these patients require repeated hospitalization which results in absenteeism and poor work performance. It imposes restriction in day to day activities due to reduced mobility and social isolation

Corresponding Author: Dr. Sukhminder Jit Singh Bajwa, House No. 27-A, Ratan Nagar, Tripuri, Patiala - 147 001, Punjab, India.

E-mail: sukhminder_bajwa2001@yahoo.com 
resulting in low self-esteem. All these factors can lead to various psychiatric diseases such as depression, anxiety, eating disorders, and many more. Sometimes, symptoms are so severe that patients had to be managed with antidepressants such as selective serotonin reuptake inhibitors (SSRIs), tricyclic antidepressants (TCAs), serotonin noradrenaline reuptake inhibitors, and even antipsychotic drugs for psychotic depression which can further interact with anti-diabetic drugs. ${ }^{[5]}$ Therefore, the present study was carried out to observe and evaluate the various psychiatric issues associated with diabetic foot ulcer or amputation, the drugs used to treat these symptoms and the resulting metabolic abnormalities produced by these drugs and their effect on diabetes itself.

Aims

The aim of the present study was to observe and evaluate various psychiatric disorders associated with diabetic foot ulcers and their treatment. Secondary outcome was to observe the effect of antidepressants, antianxiety, and antipsychotic drugs on the course of diabetes.

\section{MAterials And Methods}

In the present study, a cohort of 293 patients in the age group of $25-70$ years of either gender who visited orthopedic, surgery, anesthesia, and psychiatry department from April 1, 2011 to March 31, 2013 for the management of diabetic foot ulcers and related complications were prospectively followed over a period of 24 months. Patients were included in the study after taking informed consent. Among these, there were three dropouts. A phenomenological approach using semi-structured, conversational interviews was used to enable patients to record their experiences. They were interviewed for the type of diabetes (either type I or II), duration of diabetes and duration of development of foot ulcer and other complications, type of treatment for diabetes (either insulin or oral hypoglycemic or both) and treatment for diabetic foot (debridement or amputation). They were also asked about the psychosocial impact of foot ulceration on their daily routine activities. Throughout the interview, they were encouraged to narrate the effects of foot ulcers on their quality-of-life and their socio-behavioral attitude. International classification of diseases-10 criterion was used for diagnosis of the psychiatric disorder. The Hamilton anxiety rating scale (HAM-A) ${ }^{[6]}$ questionnaire was used to rate the severity of patient's anxiety. The scale consists of 14 items, each defined by a series of symptoms, and measures both psychic-anxiety (mental agitation and psychological distress) and somatic-anxiety (physical complaints related to anxiety). Each item is scored on a scale of $\mathrm{O}$ (not present) to 4 (severe), with a total score range of 0-56, where <17 indicates mild anxiety, 18-24 mild to moderate anxiety, and 25-30 moderate to severe anxiety. The Hamilton rating scale for depression, ${ }^{[7]}$ also called the Hamilton depression rating scale, abbreviated as HAM-D, questionnaire was used to provide an indication of depression, and as a guide to evaluate recovery. Of the 21 items, the first 17 are scored. Eight items are scored on a 5 point scale, ranging from $0=$ not present to $4=$ severe. Nine items are scored from 0 to 2 .

Statistics and data 9 (STATA 9) (Stata, College Station, TX, USA) software were used for statistical analysis. Completers and dropouts at 12 months were compared on baseline data using the t-test and $\chi^{2}$-test for homogeneity. The distribution of the main explanatory variables and potential confounders at baseline and of the outcomes were computed using percentage proportions or means \pm standard deviation. The data of the patients who were visiting psychiatric clinics for the treatment of psychiatric symptoms associated with diabetic foot ulceration were collected and analyzed for the type of psychiatric disorder, treatment options, type of drugs administered and any side effects or complications occurring as a result of these drugs.

\section{RESULTS}

In the present study, 290 participants, 183 (63.10\%) were males, and 107 (36.89\%) were females. The mean age of the patients having diabetic foot ulcer was $52.36 \pm 7.8$ years. One hundred and ninety-four $(66.89 \%)$ patients had type II diabetes and $96(33.10 \%)$ patients had type I diabetes. The mean duration of diabetes in these patients was $11.5 \pm 5.4$ years and the mean duration of development of foot ulceration after initial detection of diabetes was $4.5 \pm 3.2$ years [Table 1]. One hundred and forty-eight $(51.03 \%)$ patients were on oral hypoglycemics, $120(41.37 \%)$ patients were on insulin therapy, and 22 (7.58\%) were taking oral hypoglycemics and insulin both for control of diabetes. Among 290 patients of foot ulceration, $26(8.96 \%)$ patients had amputation at the level of knee, 14 (4.82\%) patients had above knee amputation, $23(7.93 \%)$ patients had amputation at the level of ankle, $18(6.20 \%)$ patients had amputated digits, and $209(72.06 \%)$ patients had ulcerated wound which was managed conservatively [Table 2].

Among the total participants, 40 patients (13.7\%) were already retired from their jobs but they were facing problems 
in day to day activities. Thirty-five (12.06\%) patients had to leave their jobs because of their restricted mobility and repeated hospitalization due to foot ulceration. Fifty-eight (16.55\%) participants felt that they were a burden on their family members as they were not able to earn for their family and were helpless to take care of themselves. Among them, 22 (7.5\%) participants were so disturbed that they had even lost their hope to live.

Eighty-six (29.65\%) patients were diagnosed with clinically significant psychiatric symptoms. Among them, 64 (22.06\%) patients had depression according to ICD-10 criterion. (HAM-D Scale was used for assessing the severity of depression ${ }^{[8]}$ and 22 patients $(7.58 \%)$ were diagnosed with generalized anxiety disorder in accordance to ICD-10 criterion (HAM-A scale was used for assessing the severity). ${ }^{[6]}$ Three patients (1.03\%) were diagnosed with posttraumatic stress disorder (PTSD) which developed after surgical amputation of the limb. Sixteen patients $(5.51 \%)$ had postoperative delirium after foot amputation. Delirium was more common in older patients $>50$ years of age [Table 3 ].

\begin{tabular}{lc}
\hline \multicolumn{3}{l}{ Table 1: Demographic profile of participants } \\
\hline Demographic characteristics & Numerical value \\
\hline Mean age of the patients & $52.36 \pm 7.8$ years \\
Mean duration of diabetes mellitus & $11.5 \pm 5.4$ years \\
Mean duration of foot ulceration & $4.5 \pm 3.2$ years \\
Type of diabetes (\%) & \\
Type I & $96(33.10)$ \\
Type II & $194(66.89)$ \\
Gender (\%) & \\
Male & $183(63.10)$ \\
Female & $107(36.89)$ \\
\hline
\end{tabular}

\begin{tabular}{llr}
\hline $\begin{array}{l}\text { Table 2: Management of diabetes mellitus and diabetic foot } \\
\text { ulceration }\end{array}$ \\
\hline Management & Type of treatment & $\begin{array}{c}\text { Number of } \\
\text { patients (\%) }\end{array}$ \\
\hline $\begin{array}{llr}\text { Treatment of } \\
\text { diabetes mellitus }\end{array}$ & Oral hypoglycemics & $148(51.03)$ \\
& Insulin & $120(41.37)$ \\
Management & Both & $22(7.58)$ \\
of diabetic foot & Above knee amputation & $14(4.82)$ \\
ulceration & At level of knee & $26(8.96)$ \\
& amputation & \\
& Ankle level amputation & $23(7.93)$ \\
& Digital amputation & $18(6.20)$ \\
& Conservative treatment & $209(72.06)$ \\
\hline
\end{tabular}

\begin{tabular}{lc}
\hline $\begin{array}{l}\text { Table 3: Various psychiatric disturbances in patients with } \\
\text { diabetic foot }\end{array}$ \\
\hline Type of psychiatric disorders & Number of patients (\%) \\
\hline Depression & $64(22.06)$ \\
Generalized anxiety disorders & $22(7.58)$ \\
Posttraumatic stress disorders & $3(1.03)$ \\
Postoperative delirium & $16(5.51)$ \\
Total & 105 \\
\hline
\end{tabular}

Of 86 patients diagnosed with depression and anxiety disorders, 38 patients were taking SSRIs, 20 patients were on TCAs and 16 patients were on serotonin norepinephrine reuptake inhibitors medications, and 12 patients were managed with counseling and behavior therapy. Twelve patients were also taking antipsychotics for psychotic component of depression. The data of the patients taking antidepressant and antipsychotic medications were collected to analyze the metabolic abnormalities produced by these drugs and their effect on blood sugar levels. Among 86 patients, 32 patients had poor glycemic control with glycosylated hemoglobin levels in the range of 7-9 and they were having chronic nonhealing ulcers. They had also poor response to antidepressant drugs given to treat depression. In ten patients receiving TCAs, there was uncontrolled hyperglycemia $\left(\mathrm{Hb} \mathrm{I}_{\mathrm{AC}}>9\right)$ and they were still having severe depression despite taking antidepressants.

\section{DISCUSSION}

Hyperglycemia is a primary symptom of diabetes mellitus which results either due to absolute, or relative insulin deficiency. The prevalence of depressive disorders in diabetic patients is two times more common as compared to that in the general population. ${ }^{[9]}$ In crosssectional studies, ${ }^{[10,11]}$ it was observed that depressive disorders were associated with poor glycemic control, resulting in worsening of complications attributable to diabetes. Effects of antidepressants on blood sugar levels were also comprehensively studied by Moosa et al. ${ }^{[12]}$ They followed nondiabetic depressed women using imipramine and fluoxetine for 3 months, and it was observed that in the imipramine group, an increase in body mass index (BMI), decrease in insulin secretion and increased insulin resistance was observed which resulted in hyperglycemia in nondiabetic patients. Most of the antidepressants such as nortriptyline and mirtazapine were associated with increased BMI, increased leptin concentration, and poor glycemic control. Lustman et al., also observed that those patients receiving nortriptyline for the treatment of depression for a period of 8 weeks or more, were having poor glycemic control with worsening of diabetes. ${ }^{[13,14]}$

In the present study, of 290 patients, 86 patients were having psychiatric disorders and out of them 64 $(22.06 \%)$ patients were suffering from depression. After the interview, it was revealed that most of the participants after developing foot ulceration were 
forced to lead a restricted life. They were not able to carry out their day to day activities as they were doing it before their disability like self-care, household chores, and sports-related activities. With the restriction in daily activities, they were not able to lead a healthy social life which resulted in social isolation. Ismail et al., ${ }^{[15]}$ had observed that one-third of patients having diabetic foot ulcer suffered from clinically significant depression during their lifetime. In our study, patients of younger age group, that is, $<45$ years were more depressed as compared to older patients. Those patients suffering from severe psychotic depression were having comparatively larger ulcers, and they also had poor social support. This could be explained by the fact that due to larger ulcers, patients were not able to carry out daily activities which lead to depression and with poor support system they were not able to take care of their wound which further caused the clinical deterioration of their ulcer creating a vicious cycle. Patients with severe depressive disorder were not adhering to their dietary control and modifiable lifestyle changes which further aggravated the socioclinical situation as compared to those patients who were not having severe depression. Carrington et al. ${ }^{[16]}$ observed that people with chronic foot ulceration had significantly more symptoms of depression than patients without foot ulceration, and these symptoms were associated with impaired wound healing. A study reported that various problems faced by patients as a result of foot ulceration might result in feelings of depression. Depression resulted in decreased self-care which further aggravated the ulcers. This suggests a bi-directional relation between depression and diabetic foot ulcer. ${ }^{[17]}$

Therefore, it is very important to take care of the psychosocial aspect of the illness, one important aspect of which is awareness and counseling about the illness, second is its medical management. In our study, it was observed that 12 patients were aware of their illness and were better-prepared to cope with it. They were managed with counseling alone. Counseling of both patients and family members is very important in complicated cases such as those admitted in intensive care unit for diabetic complication..$^{[5]}$ There is a need to educate the people in their own language about various aspects of the course of illness. The medical and nonmedical personnel should be sensitized about the harmful effects of uncontrolled diabetes and its implications, so that they can convey it to the patients and their families in an appropriate manner. ${ }^{[18]}$
In this study, 32 patients on antidepressants had poor glycemic control and were not responding to insulin. In a study done by Kivimäki et al., it was concluded that with the use of antidepressants (SSRIS and TCAs), there is increased chances of developing type 2 diabetes. The 5 -year risk of developing diabetes increased in a dose-response fashion, depending on the level of exposure to antidepressant medication. ${ }^{[19]}$ Frank Anderson et al., also concluded that long-term use of antidepressants (SSRIs and TCAs) were associated with increased risk of diabetes. ${ }^{[20]}$ Due to severe depression, patients were nonadherent to treatment regimens for diabetes which further deteriorated their foot ulcers, as well as significantly impaired wound healing. Monami et al. ${ }^{[21]}$ observed that diabetic foot ulcer patients of 60 years or older with geriatric depression scale (GDS) scores of $>10$ had significantly higher risk of nonhealing ulcers. Adherence, as measured by the percent of patients wearing prescribed footwear at follow-up, was significantly lower among patients with a GDS score over 10.

In the present study, 22 (7.58\%) patients were having generalized anxiety disorder. Majority of these patients had nonhealing recurrent ulcers. Ragnarson Tennvall and Apelqvist ${ }^{[22]}$ observed that symptoms of anxiety were less in patients with primary healed ulcers as compared to patients having recurrent nonhealing foot ulcers. Complications of diabetes have been shown to be the most important predictors for anxiety symptoms among people with diabetes. ${ }^{[23,24]}$ Carrington et al., ${ }^{[16]}$ however, in their study did not find any difference in the symptoms of anxiety, in patients with diabetic foot ulceration or amputated limb as compared to patients having diabetes alone.

Studies have shown that mood or anxiety disorder further exacerbates the patient's already impaired wound healing. Due to anxiety, there is stress-induced increase in glucocorticoid levels which further suppresses interleukin-1 B (IL-1B), tumor necrosis factor and platelet-derived growth factor. IL-1 beta regulates the production, release and activation of metalloproteinases, which play an important role in the formation of the connective tissue matrix required for healing. ${ }^{[25]}$ Broadbent studied the effect of stress on surgical wound healing by analyzing wound fluid for the levels of specific cytokines. There were low levels of IL-1 and matrix metallopeptidase 9, indicating that stress impairs the inflammatory responses and matrix degradation processes that one sees in the early stages of normal wound healing. Thus, poor wound healing will further worsen the foot ulceration leading to more psychological disturbance" ${ }^{[26]}$

In the present study, 12 patients were having psychotic symptoms along with depression. Previous literature 
Neeru, et al.: Psychosocial and clinical aspects of diabetic foot

suggests that in addition to increased incidence of anxiety and depression, patients with diabetes also face cognitive and behavioral impairments. This might be due to the effect of prolonged hyperglycemia on the brain. In addition, oxidative stress and reactive oxygen species are increased in patients with diabetes and both reduce the activity of a variety of proteins that are crucial to neuronal homeostasis. ${ }^{[3]}$

Post-traumatic stress disorder was seen only in $3.70 \%$ of the patients who had undergone surgical amputation. It was already reported that PTSD is relatively rare $(<5 \%)$ among patients who had undergone amputation following a chronic illness like diabetes as compared to amputation after accidental injury ${ }^{[4]} \mathrm{A}$ meta-analysis demonstrated an association between hyperglycemia and anxiety disorder in type 1 and type 2 diabetes mellitus. ${ }^{[25]}$ and it was concluded that "Although the association between anxiety and diabetes-related complications may be shown in various studies in hospital based setting, not much was known about the prevalence of anxiety symptoms in people with diabetic foot ulcer in community-based samples". [17] Diabetic patients and their family members should be sensitized about the implications of diabetes mellitus. Awareness and counseling about illness should be started at an early stage so that the use of antidepressant drugs for treating depression could be avoided.

\section{CONCLUSION}

It was concluded that diabetic foot ulcer patients had increased prevalence of psychiatric disorders, depression being more common. Anti-depressant and anti-psychotic drugs used to treat psychiatric symptoms further lead to hyperglycemia and worsening of foot ulceration. Thus, a vicious cycle developed, foot ulceration lead to depression, depression lead to poor self-care and with the use of antidepressants, diabetes worsened further.

\section{REFERENCES}

1. Shahi SK, Kumar A, Kumar S, Singh SK, Gupta SK, Singh TB. Prevalence of diabetic foot ulcer and associated risk factors in diabetic patients from North India. J Diabet Foot Complications 2012;4:83-91.

2. Kumar S, Ashe HA, Parnell LN, Fernando DJ, Tsigos C, Young RJ, etal. The prevalence of foot ulceration and its correlates in type 2 diabetic patients: A population-based study. Diabet Med 1994;11:480-4.

3. Scott G. The diabetic foot examination: A positive step in the prevention of diabetic foot ulcers and amputation. Osteopath Fam Physician 2013;5:73-8.

4. Diabetes foot care: step-by-step. Available from: http:// www.worlddiabetesfoundation.org/projects/India TanzaniaWDF03-056. [Last accessed on 2014 Jun 05].

5. Bajwa SS, Sehgal V. Psycho-social and clinical aspects of diabetocriticare. J Soc Health Diabet 2013;1:70-4.
6. Hamilton M. The assessment of anxiety states by rating. Br J Med Psychol 1959;32:50-5.

7. Hamilton Depression Scale. Available from: http://www. psychcongress.com/saundras-corner/scales-screeners/ depression/hamilton-depression-rating-scale-ham-d. [Last accessed on 2014 Jun 05].

8. Hedlund JL, Viewing BW. The Hamilton rating scale for depression. J oper psychiatry 1979;10:149-65.

9. Anderson RJ, Freedland KE, Clouse RE, Lustman PJ. The prevalence of comorbid depression in adults with diabetes: A meta-analysis. Diabetes Care 2001;24:1069-78.

10. Lustman PJ, Anderson RJ, Freedland KE, de Groot M, Carney RM, Clouse RE. Depression and poor glycemic control: A meta-analytic review of the literature. Diabetes Care 2000;23:934-42.

11. de Groot M, Anderson R, Freedland KE, Clouse RE, Lustman PJ. Association of depression and diabetes complications: A metaanalysis. Psychosom Med 2001;63:619-30.

12. Moosa MY, Panz VR, Jeenah FY, Joffe BI. African women with depression: The effect of imipramine and fluoxetine on body mass index and leptin secretion. J Clin Psychopharmacol 2003;23:549-52.

13. Lustman PJ, Griffith LS, Clouse RE, Freedland KE, Eisen SA, Rubin $\mathrm{EH}$, et al. Effects of nortriptyline on depression and glycemic control in diabetes: Results of a double-blind, placebocontrolled trial. Psychosom Med 1997;59:241-50.

14. Laimer M, Kramer-Reinstadler K, Rauchenzauner M, Lechner-Schoner T, Strauss R, Engl J, et al. Effect of mirtazapine treatment on body composition and metabolism. J Clin Psychiatry 2006;67:421-4.

15. Ismail K, Winkley K, Stahl D, Chalder T, Edmonds M. A cohort study of people with diabetes and their first foot ulcer. The role of depression on mortality. Published ahead of print at. Available from: http://www.care.diabetesjournals.org on 15 March 2007. DOI: 10.2337/dc06-2313. [Last accessed on 2014 Jun 05].

16. Carrington AL, Mawdsley SK, Morley M, Kincey J, Boulton AJ. Psychological status of diabetic people with or without lower limb disability. Diabetes Res Clin Pract 1996;32:19-25.

17. An Epidemiologic Study of Diabetes-Related Foot Ulcers Issues Related to Prevention and Mortality Based on the Nord-Trøndelag Health Study HUNT Marjolein Memelinklversen Department of Public Health and Primary Health Care, Section of Nursing Sciences. Norway: University of Bergen; https://bora.uib.no/.../ Dr.thesis_Marjolein\%20M.\%20lversen.pdf [Last accessed on 2014 Jun 05].

18. Bajwa SJ, Kalra S. Diabeto-anaesthesia: A subspecialty needing endocrine introspection. Indian J Anaesth 2012;56:513-7.

19. Kivimäki M, Hamer M, Batty GD, Geddes JR, Tabak AG, Pentti J, et al. Antidepressant medication use, weight gain, and risk of type 2 diabetes: A population-based study. Diabetes Care 2010;33:2611-6.

20. Andersohn F, Schade R, Suissa S, Garbe E. Long-term use of antidepressants for depressive disorders and the risk of diabetes mellitus. Am J Psychiatry 2009;166:591-8.

21. Monami M, Longo R, Desideri CM, Masotti G, Marchionni N, Mannucci E. The diabetic person beyond a foot ulcer: Healing, recurrence, and depressive symptoms. J Am Podiatr Med Assoc 2008;98:130-6.

22. Ragnarson Tennvall G, Apelqvist J. Health-related quality of life in patients with diabetes mellitus and foot ulcers. J Diabetes Complications 2000;14:235-41.

23. Collins MM, Corcoran P, Perry IJ. Anxiety and depression symptoms in patients with diabetes. Diabet Med 2009;26:153-61.

24. Peyrot M, Rubin RR. Levels and risks of depression and anxiety symptomatology among diabetic adults. Diabetes Care 1997;20:585-90. 
25. Anderson RJ, Grigsby $A B$, Freedland $K E$, de Groot $M$, McGill JB, Clouse RE, et al. Anxiety and poor glycemic control: A meta-analytic review of the literature. Int J Psychiatry Med 2002;32:235-47.

26. Nancy R. Megas MSN, CRNP: How Depression and anxiety affect patient adherence. Available from: http://www.podiatrytoday. com/how-depression-and-anxiety-affect-patient-adherence. [Last accessed on 2014 Jun 05].
How to cite this article: Neeru B, Gagandeep K, Pal AJ, Bajwa SS, Harbandna S, Rajesh K. Psychosocial, psychiatric, and clinical implications of diabetic foot ulceration: A prospective analysis. J Soc Health Diabetes 2015;3:89-94.

Source of Support: Nil. Conflict of Interest: None declared. 